\title{
De la farmacia a la toxicología. La química en tribunales y la conformación de una experticia judicial, Santiago de Chile, 1831-1897
}

Resumen: Este artículo introduce al desarrollo de la experticia química en Santiago de Chile a través de los peritos que participaron como auxiliares de la justicia en casos de envenenamiento entre 1831 y 1897 . Se propone identificar a estos expertos e indagar en la relación que establecieron con ciertas áreas del conocimiento -específicamente la farmacia y la toxicología - con el objeto de cruzar su experiencia laboral o disciplinar con la conformación de su experticia en tribunales. En paralelo indaga la relación entre Estado y ciencia desde la tribuna particular otorgada por el aparato judicial. Palabras clave: química, farmacia, toxicología, justicia, envenenamiento.

\section{From Pharmacology to Toxicology. Chemistry in the courtroom and the development of a judicial expertise, Santiago de Chile, 1831-1897}

Abstract: This article explores the development of chemical skills in Santiago de Chile through the judicial experts that participated in poisoning cases between 1831 and 1897. It aims to identify these experts and to investigate their relationship with certain knowledges -specifically pharmacy and toxicology-, in order to connect their work and disciplinary experience, with the conformation of their judicial expertise. Additionally, the study focusses on the relationship between science and the State, from the particular perspective of the judicial apparatus.

Keywords: chemistry, pharmacy, toxicology, poisoning.

\section{Da farmácia à toxicologia. A química nos tribunais e a conformação de uma expertise judicial, Santiago do Chile, 1831-1897}

Resumo: Este artigo introduz ao desenvolvimento da expertise química em Santiago do Chile através dos peritos que participaram como auxiliares da justiça em casos de envenenamento, entre 1831 e 1897. Propõe-se identificar esses especialistas e indagar na relação que estabeleceram com certas áreas do conhecimento- especificamente farmácia e toxicologia- a fim de cruzar sua experiência de trabalho ou disciplinar com a conformação de sua expertise em tribunais. Ao mesmo tempo, indaga-se na relação entre Estado e ciência, a partir da tribuna particular dada pelo aparato judiciário.

Palavras-chave: química, farmácia, toxicologia, justiça, envenenamento.

Cómo citar este artículo: María José Correa Gómez, "De la farmacia a la toxicología. La química en tribunales y la conformación de una experticia judicial, Santiago de Chile, 1831-1897”, Trashumante. Revista Americana de Historia Social 15 [2020]: 58-81.

DOI: 10.17533/udea.trahs.n15a03

Fecha de recepción: 21 de octubre de 2018

Fecha de aprobación: 9 de mayo de 2019

María José Correa Gómez: Doctora en Historia por University College London. Profesora del Departamento de Humanidades, Universidad Andrés Bello.

Correo electrónico: maria.correa@unab.cl

. 


\title{
De la farmacia a la toxicología. La química en tribunales y la conformación de una experticia judicial, Santiago de Chile, 1831-1897
}

\author{
María José Correa Gómez
}

\begin{abstract}
A mediados del siglo XIX, durante el proceso de constitución de la estructura disciplinaria moderna de las ciencias, la química comenzó a adquirir un contorno y una posición específica que la legitimó como disciplina universitaria y como saber de Estado. Este artículo revisa parte de este proceso desde la química forense, y propone que la realización de análisis en el contexto judicial y su capacidad resolutiva incidieron en su definición y reconocimiento. Sugiere que el estudio de los expertos que participaron en dichos procesos forenses - boticarios, farmacéuticos y toxicólogos - y su validación como parte de las élites técnicas que prestaron servicios al Estado permiten conocer no solo la experticia química, sino también procesos relevantes expresados en la ciencia y la sociedad. ${ }^{1}$ Así, entendiendo la intervención de los expertos en el entramado estatal como un hecho fundamental de la transformación de sus quehaceres en ejercicios legítimos y monopólicos, en términos prácticos y cognitivos, el estudio propone seguir sus recorridos judiciales y a través de ellos delinear algunos aspectos del desarrollo, visibilidad y legitimidad de la química. ${ }^{2}$

Lo científico se sostuvo en el Estado, en su soporte organizativo y burocrático, en sus programas y necesidades. La historiografia ha mostrado cómo el adelanto o el abandono, así como la definición y el desdibujo de ciertas disciplinas, se anudaron a las agendas de gobierno, pero también ha advertido el entrecruzamiento de estas agendas con las dinámicas cotidianas y los intereses particulares de los expertos y de

* Se agradece a Nicolás Araya por su colaboración en el trabajo de archivo, a los evaluadores por sus pertinentes comentarios y a los asistentes al Laboratorio de Historia de la Ciencia,Tecnología y Sociedad, instancia en donde fue presentado un adelanto de esta publicación. Esta investigación cuenta con el financiamiento del proyecto Fondecyt Iniciación número 11150340 (2015-2019).

1. Para el estudio de la ciencia y lo público, véase Jan Golinski, Science as Public Culture. Chemistry and Enlightenment in Britain, 1760-1820 (Cambridge: Cambridge University Press, 1999).

2. Ricardo González, "Itinerarios de la profesión médica y sus saberes de Estado. Buenos Aires, 1850-1910", Los saberes del Estado, coords. Mariano Ben Plotkin y Eduardo Zimmermann (Buenos Aires: Edhasa, 2012) 125-152; Eric Ash, "Introduction: Expertise and the Early Modern State", Osiris 25.1 (2010): 1-24.
\end{abstract}


quienes se intersecaron con el saber aplicado. ${ }^{3}$ Así, si bien el desarrollo de la ciencia se vinculó con los destinos de la nación a través de diversos establecimientos e iniciativas, también se intercaló con los recorridos seguidos por los científicos, expertos o conocedores en su quehacer diario en dichas instituciones. ${ }^{4}$

Este estudio se centra en esa relación y en esos recorridos. Propone que el uso de la química en cuestiones judiciales asociadas a venenos contribuyó en la definición y reconocimiento de la llamada "farmacia legal" y participó, junto a otras experiencias científicas, en el desarrollo de la química. En este sentido, lo interesante de la justicia es que, gracias a la urgencia y sonoridad del crimen, la química, la farmacia legal y sus especialistas alcanzaron una constancia y una visibilidad diferente a la que tenían en otros ámbitos de su quehacer que, si bien no la ubicó como área privilegiada en el ámbito científico, sí le confirió una estabilidad y una proyección interesante de estudiar.

Así, por medio del seguimiento a los recorridos de Vicente Bustillos, boticario, farmacéutico y precursor de la farmacia nacional, y Pedro Lemetayer, toxicólogo a la cabeza del laboratorio del Instituto de Higiene, en procesos judiciales por envenenamiento entre 1831 y 1897 , el estudio revisa el progreso de la pericia química en su función rectora como herramienta para leer la ciudad y comprender lo invisible. Propone que el aporte de estos expertos en la administración estatal, específicamente en la justicia civil y criminal, en juicios de adulteraciones, falsificaciones, suicidios, homicidios y envenenamientos contribuyó a una mayor "versación en las manipulaciones químicas" y al reconocimiento del valor de este quehacer científico en la sociedad. ${ }^{5}$

Seguir el trayecto desplegado por los propios peritos permite trabajar con las subjetividades, intereses y circunstancias que influyeron en la pericia química y que le fueron dando protagonismo a distintos actores, como es el caso de los

3. Jan Golinski, Making Natural Knowledge. Constructivism and the History of Science (Chicago: The University of Chicago Press, 2005) 47-78; Steven Shapin, Never Pure. Historical Studies of Science as if It Was Produced by People with Bodies, Situated in Time, Space, Culture, and Society, and Struggling for Credibility and Authority (Baltimore:The John Hopkins University Press, 2010) 212-233; Ernesto Bohoslavsky y Germán Soprano, eds., Un Estado con rostro humano. Funcionarios e instituciones estatales en Argentina (desde 1880 a la actualidad) (Buenos Aires: Prometeo Libros, 2010) 9-55; Mariano Ben Plotkin y Eduardo Zimmermann, "Introducción. Saberes de Estado en la Argentina, siglos XIX y XX", Los saberes del Estado, coords. Mariano Ben Plotkin y Eduardo Zimmermann (Buenos Aires: Edhasa, 2012) 17.

4. Espacios como el Instituto Nacional (1813), el Museo Nacional (1830), la Universidad de Chile (1833), el Instituto Nacional de Estadísticas (1843), el Instituto Pedagógico de Santiago (1889), el Instituto de Higiene (1892) o los mismos tribunales. Carlos Sanhueza, "El objetivo del Instituto Pedagógico no es el de formar geógrafos. Hans Steffen y la transferencia del saber geográfico alemán a Chile. 1893-1907”, Historia 45.1 (2012): 171-197; Carlos Sanhueza, “Objetos naturales en movimiento. Acerca de la formación de las colecciones del Museo Nacional de Chile (18531897)", Revista de Humanidades 34 (2016): 143-169; Carlos Sanhueza, Geografía en acción. Práctica disciplinaria de Hans Steffen en Chile (1889-1913) (Santiago: Editorial Universitaria, 2014).

5. Federico Puga Borne, Compendio de Medicina Legal adaptado a la legislación chilena (Santiago: Imprenta Cervantes, 1896) 9. 
médicos. ${ }^{6}$ Estos nuevos especialistas fueron en su mayoría egresados de farmacia que complementaron la labor fármaco-legal realizada previamente por los facultativos, a quienes reemplazaron gradualmente en aquellas tareas que requerían de la química. Como resultado, los farmacéuticos comenzaron a posicionarse como especialistas del ámbito químico más allá de su labor en las boticas como preparadores de medicinas, y ampliaron las definiciones de sus quehaceres y saberes. Con el tiempo su contribución judicial alcanzó mayor precisión incorporando nuevas áreas como la toxicología.

Pese a que no es novedad el uso de la justicia y de sus registros en la historia de la ciencia, esta entrada no ha sido mayormente trabajada para el caso chileno desde el ámbito de la química. ${ }^{7}$ Las investigaciones que se han realizado en los últimos años se centraron en el accionar pericial vinculado a la definición de la capacidad y la voluntad, con lo cual se dio cuenta del aporte de las fuentes judiciales para la historia de la ciencia y, particularmente, el estudio de las prácticas científicas. ${ }^{8}$

En esta dirección, el estudio intenta responder ¿quiénes fueron estos expertos? ¿De dónde venían? ¿Qué sabían y qué podían hacer? ¿Cómo fueron definidos y presentados? ¿Qué podían ofrecer al Estado y a la justicia? ¿Qué tipo de conocimientos, experiencias y recursos apoyaron su identidad de conocedores? ¿Cómo se expresó su autoridad en tribunales? ¿Qué variabilidad mostró su competencia forense y qué tránsitos se identifican en ella? ¿Cómo valoraron su capacidad? De esta manera, la ganancia de una determinada aptitud, la evaluación de su manejo, la diferencia entre el uso ligero de ciertos conocimientos y la adquisición de una capacidad orientada a la creación de sentido, los modos de determinación de un saber avezado, su uso y credibilidad, sus formas de implementación y sus riesgos son algunas de las varias cuestiones que se buscan analizar, con la intención de profundizar en el aporte de la química a la justicia, en la transformación del ámbito forense desde el arte boticario a la toxicología y en el carácter de la competencia exhibida por los expertos.

6. Sobre autopsias véase Mario Fabregat, "Justicia y procedimientos judiciales por suicidio e intento de suicidio. Chile: 1820-1920" (Tesis de doctorado en Historia, Universidad de Chile, 2017).

7. Los trabajos señeros del ámbito internacional se han enfocado en el cruce enfermedad-crimen, como Nigel Walker, Crime and Insanity in England (Edinburgh: University Press Edinburgh, 1968); Roger Smith, Trial by Medicine. Insanity and Responsibility in Victorian Trials (Edinburgh: Edinburgh University Press, 1981); Joel Peter Eigen, Witnessing Insanity. Madness and Mad-Doctors in the English Court (New Haven:Yale University Press, 1995).

8. Víctor Brangier, "La fragilidad de la simbiosis médico-judicial y la producción de una antropología criminal. Juzgados del Crimen de Santiago, 1874-1906" (Tesis de maestría en Historia, Universidad de Chile, 2008); Mariano Ruperthuz Honorato y Marcelo Sánchez Delgado, "Entre la degeneración y el psicoanálisis: una pericia médico legal chilena en 1915”, Revista Historia y Justicia 4 (2015): 138168; María José Correa, "De la Casa de Orates al juzgado. Pericia alienista y evaluación judicial de la locura en Santiago de Chile hacia 1860", História, Ciências, Saúde - Manguinhos 20.2 (2013): 571-585; Silvana Vetö,"Homosexualidad masculina y examinación médica en la Casa de Menores de Santiago, 1929-1942”, Revista Historia y Justicia 10 (2018): 42-76. 


\section{Bustillos, un boticario en tribunales. Santiago, 1831}

En octubre de 1831 Juana Chacotal confesó haber puesto veneno en la bebida de su patrona, el cual había sido entregado por un boticario de calle Ahumada, quien sabiendo que "le daban muy mal trato en su casa" había confesado que "le había dado aquello para que se librara de sus padecimientos". ${ }^{9}$ La justicia manejó varias opciones sobre la identidad de la sustancia ofrecida. Inicialmente se pensó que la sustancia era solimán y luego carbonato de potasio, ${ }^{10}$ lo que fue contradicho por el "profesor Bustillos", quién determinó que correspondía a "sulfato de zinc". ${ }^{11}$ A esta identificación preliminar le siguió un informe emitido por el facultativo Tomás Armstrong "en consorcio con el profesor de Farmacia Don José Bustillos" para conocer la calidad y propiedad del sulfato, la dosis en que actuaba como veneno y la cantidad añadida a la bebida preparada por Juana. En un primer mensaje Armstrong, británico vinculado a la enseñanza de la medicina, explicó que para informar debía examinar primero a la afectada y conocer sus síntomas, "no siendo de ningún modo suficiente en medicina legal las pruebas manifestadas por el profesor de farmacia, para poder en juicio asegurar con evidencia exclusiva el sulfato de zinc, en el líquido supuestamente envenenado", para lo que apelaba al juicio clínico que determinaba su oficio. ${ }^{12} \mathrm{~A}$ esta réplica siguió un informe pericial firmado por Armstrong y Bustillos que, pese a respaldar la hipótesis inicial del farmacéutico, develó la recepción ambivalente que tuvo la apreciación del boticario, la que necesitó de la compañía de un médico para validarse.

En 1831, y a sus 31 años, José Vicente Bustillos y Maceira (1800-1873) era un respetable conocedor de la farmacia. ${ }^{13}$ En un contexto como el chileno y en un momento de organización de las ciencias, ya era considerado una autoridad en el ámbito científico. Había forjado su posición en un escenario mixto que integraba los estudios esporádicos realizados en el Instituto Nacional — donde habría mostrado interés por la química y la física bajo la influencia del presbítero José Alejo Bezanilla y del otrora ensayador de la Casa de Moneda, Francisco Rodríguez Brochero - con el trabajo de botica, la enseñanza y la administración pública de la química. En sus estudios se acercó a la enseñanza formal de la ciencia y en su trabajo en la botica de Nathaniel Cox "encontró un magnífico laboratorio en el cual podía hacer sus estudios esperimentales i todo jénero de investigaciones analíticas de que se ocupa aquella ciencia". ${ }^{14}$ En paralelo comenzó "la carrera del profesorado",

9. “Criminal contra Juana por haberle dado veneno a su señora”, Santiago, 1831. ANH, Santiago, Archivo Judicial de Santiago, legajo 1605, exp. 1, f. 2.

10. Véase Paula Ronderos, "El arte de boticario durante la primera mitad del siglo XVII en el Nuevo Reino de Granada", Fronteras de la Historia 12 (2007): 184.

11. "Criminal contra Juana”, f. 2.

12. "Criminal contra Juana", f. $3 \mathrm{v}$.

13. Óscar Aedo, José Vicente Bustillos: padre de la farmacia chilena (Santiago: Academia de Ciencias Farmacéuticas de Chile, 1987).

14. En 1827 dicha botica pasó a estar bajo su tutela. Resultaba interesante que ese traspaso fuese 
asignándosele la nueva clase de "química orgánica aplicada a la medicina i farmacia" en el Instituto Nacional. ${ }^{15}$ Junto con su incursión en la botica y en la enseñanza, Bustillos encontró un lugar entre las autoridades científicas del periodo, cuando en 1830 se reestableció el Protomedicato y se lo nombró profesor de Farmacia del Tribunal. ${ }^{16} \mathrm{Su}$ perfil de boticario, profesor y autoridad respaldaba su presencia en la pericia y, posiblemente, lo presentaba como el conocedor de química más idóneo para realizar la indagación. Esto ocurría en un momento en el que las leyes republicanas aún no ordenaban los procesos probatorios de los juicios, con lo que persistía la influencia de regulaciones coloniales que habían tendido a convocar a los médicos en los procesos de envenenamiento, ${ }^{17}$ vinculados para algunos ámbitos del espacio colonial americano a la criminalización de la yerbatería. ${ }^{18}$

A falta de un marco legal definido, los expertos en el área de la química susceptibles de ser llamados por los jueces a asistir en este tipo de juicios se vincularon con los criterios de experticia mostrados por Bustillos, al menos para la ciudad de Santiago. El propio Bustillos, considerado a su temprana edad como uno "de los chilenos más ilustrados en ciencias físicas y naturales", designado por esos años como parte de la comisión que debía evaluar el cumplimiento del contrato de Claudio Gay en Chile por sus conocimientos en farmacia y en botánica, y también por su condición de autoridad protomedical, no contaba con una certificación universitaria. ${ }^{19}$ Como plantea Patience Schell, los naturalistas extranjeros y chilenos carecían de una formación científica oficial y habían forjado sus condiciones en el trabajo de campo. Figuras como Gay y el propio Bustillos constituían referentes de un ambiente científico variado y en delimitación, que comenzaría a

interpretado como un acto que le permitió "seguir dedicado con mayor comodidad i más recursos a sus trabajos científicos". Diego Torres, "Elogio del doctor don José Vicente Bustillo: reseña de la enseñanza de la física i química en Chile. Discurso leído por don Diego A. Torres en el acto de su incorporación a la Facultad de ciencias físicas i matemáticas, en sesión de 11 de abril de 1874", Anales de la Universidad de Chile (Santiago) abril de 1874: 285.

15. Torres 286.

16. El Tribunal del Protomedicato estaba integrado por Guillermo Blest, presidente y profesor de medicina; Nataniel Cox, profesor de cirugía; Vicente Bustillos, profesor de farmacia; Pedro Morán, secretario, y José Barrios, fiscal. Su reorganización llevó a que entre 1819 y 1826 Francisco Fernández ejerciera el rol de examinador de farmacia. Su reemplazo en 1826 por la Sociedad Médica, la que a su vez fue substituida en 1827 por la Inspección General de Medicina, puso al doctor Juan Miguél como inspector de farmacia. El restablecimiento del Protomedicato en 1830 ubicó a Bustillos como máxima autoridad de farmacia, calidad que fue compartida con su ingreso a la Escuela de Medicina como profesor de Química y Farmacia en 1833.

17. La Novísima Recopilación de las Leyes de España, publicada en 1805 , ordenaba el quehacer del boticario.

18. Juan Sebastián Ariza, "La cocina de los venenos. Aspectos de la criminalidad en el Nuevo Reino de Granada, siglos XVII-XVIII" (Tesis de pregrado en Historia, Universidad del Rosario, 2013); Juan Sebastián Ariza, “¿Remedios o ponzoñas? Aproximación al uso de la yerbatería como método curativo en el Nuevo Reino de Granada durante el siglo XVIII", Anuario de Historia Regional $y$ de las Fronteras 19.2 (2014): 315-333.

19. Diego Barros Arana, Estudios histórico-bibliográficos (Santiago: Imprenta Cervantes, 1909-1911) 282. 
dibujar en el transcurso del siglo una línea cada vez más marcada, pero no por ello aclaratoria, entre expertos y profanos. ${ }^{20}$

La presencia de una figura como Bustillos en tribunales dejaba ver, entre líneas, los distintos criterios que operaban en la definición e identificación de los peritos en química. Mostraba los lazos que la pericia química comenzaba a construir con los conocedores de la farmacia en desmedro de los médicos, y con lo cual se daba cuenta de que esta última no se remitía solo a la preparación de medicinas y a la atención terapéutica — no aprobada por las autoridades—- sino a otras experiencias. Si bien la vivencia acumulada por Bustillos superaba ampliamente el ámbito de la farmacia, su elección como perito, al igual que la de otros expertos, mostró el reconocimiento que comenzaba a tener su saber en farmacia. En segundo lugar, mostraba que la botica, presentada desde hacía tiempo como un importante sitio formativo, ${ }^{21}$ seguía siendo vista como un espacio de ciencia, pero que requería ya de nuevos aportes para su validación, que ayudaran a compensar el aspecto "anticuado" asignado a los boticarios del periodo. Tal como describiría la viajera María Graham, los boticarios hacían pensar más en un herbolario "del siglo XIV”, pero en ocasiones revelaban ese "cierto gusto por la historia natural" que lo acercaba a los naturalistas decimonónicos. ${ }^{22}$

A inicios de la década de los treinta solo un grupo muy pequeño de hombres cumplía con los criterios de experticia mostrados por Bustillos, considerando que, además, Santiago contaba solo con unos pocos boticarios reconocibles que no compartían la experiencia de profesor de farmacia y autoridad pública. ${ }^{23}$ En este sentido, Bustillos se elevaba como una autoridad pericial viable entre un grupo magro de posibles especialistas; representaba no tanto lo disponible, sino lo deseable. Así, y con el reconocimiento de su ubicación dentro de la pequeña élite científica de la primera mitad del siglo XIX y su identificación como un potencial candidato para la justicia, su autoridad resumió la potestad de un personaje en tránsito que reunía la costumbre apotecaria con los criterios modernos de la química, los cuales ofrecía al servicio de las autoridades y de la propia visibilización de la farmacia. ${ }^{24} \mathrm{Su}$ tímida presencia en tribunales, en los que aún no desplegaba del todo los recursos científicos usados para leer e interpretar las pruebas presentadas a la justicia, daba cuenta del inicio de un reconocimiento que se traduciría en la identificación de la farmacia como una ciencia útil a los intereses judiciales de

20. Patience Schell, The Sociable Sciences. Darwin and His Contemporaries in Chile (New York: Palgrave Macmillan, 2013) 36 y 40.

21. Enrique Laval, Botica de los jesuitas de Santiago (Santiago: Asociación Chilena de Asistencia Social, 1953).

22. María Graham, Diario de su residencia en Chile (1822) y de su viaje al Brasil (1823) (Madrid: Editorial Americana, 1964) 176.

23. Fernando Urizar, Repertorio chileno (Santiago: Imprenta Araucana, 1835).

24. Fernando Franulic, “'Deber entrar a medicinarse...': la casa, el adentro y la purificación. El Hospital San Francisco de Borja entre 1830 y 1870" (Tesis de maestría en Historia, Universidad de Chile, 2007) 66. 
la nueva república y adecuada para la comprensión del crimen y la rearticulación del orden social a través de los tribunales. Al mismo tiempo esta magra presencia los definía como un recurso escaso y en proceso de conformación, anudada a una figura tipo: la del boticario reconocido y encumbrado, sostenido aún en su vinculación con la preparación de medicamentos, cruzado por el Protomedicato y por la nueva institucionalidad educativa que surgía en el Chile independiente.

\section{Bustillos y la Sociedad de Farmacia en tribunales. Santiago, 1850}

El 25 de abril de 1850 el médico Ildefonso Raventos informó al intendente de Santiago que tenía "fundadas sospechas" que tres enfermos que había atendido en la Calle de las Monjas Rosas habían sido envenenados. Su duda abrió una indagación destinada a descubrir a los autores y cómplices del supuesto delito, que convocó al propio Raventos y a los médicos Juan Miquel ${ }^{25}$ y Juan Mackenna para que informaran en calidad de peritos. ${ }^{26}$ Miquel identificó en los enfermos síntomas de intoxicación y reconoció en los dulces consumidos un carácter venenoso, "teniendo a la vista uno de los mencionados pastelitos, y examinado con cuidado, me pareció conocer una substancia venenosa que es conocida con el nombre de oropimente, por el color amarillo". ${ }^{27}$ Advirtiendo que su examen era superficial, en cuanto que se basaba en signos clínicos y en el "color amarillo" de los caramelos, sugirió que los eventos merecían ser evaluados por personas más versadas, como "el catedrático de química", y analizados en espacios apropiados, como el Laboratorio del Instituto Nacional. Solo así se podría confirmar el carácter tóxico de los dulces y la posible presencia de sulfato de arsénico o bromuro de plomo. ${ }^{28}$

El juez convocó a Vicente Bustillos e Isidoro Salinas para que practicaran el “análisis químico analítico" e informaran si los alimentos contenían alguna sustancia nociva capaz de producir efectos mortales. Si bien este auxilio pericial parecía derivar del consejo de Miquel, respondía en lo general a un contexto que avalaba esta sugerencia. Primero, por la paulatina modernización del aparato judicial y el establecimiento de procesos y protocolos, y segundo, por la gradual profesionalización de los farmacéuticos y su reconocimiento como ensayadores capaces de descubrir por medio del laboratorio aquellos elementos invisibles a los demás, ya alejados de la tutela médica. ${ }^{29}$

25. Se modificó la ortografia del expediente de Mikel a Miquel, respetando el apellido indicado en otras fuentes.

26. "Proceso indagatorio instruido de oficio para descubrir los autores y complices del envenenamiento hecho en la persona de Dn Wenceslao del Pozo y otros", Santiago, 1850. ANH, Santiago, Archivo Judicial de Santiago, legajo 1626, exp. 4.

27. "Proceso indagatorio", f. 8.

28. "Proceso indagatorio", f. 8.

29. El término ensayador tiene varias acepciones. Para el siglo XIX la Real Academia Española lo define como "el que tiene por oficio ensayar los metales preciosos", y también como el "que prueba, ensaya, califica y examina las cosas". En Chile encontramos en uso este término tanto para conocedores 
El llamado a Bustillos y Salinas ratificaba este contexto y confirmaba el protagonismo de la farmacia como disciplina principal de la pericia en casos de venenos. Por una parte, Bustillos había sumado para 1851 nuevos reconocimientos que lo ubicaban ya no solo como un científico avezado, sino como uno de los fundadores de la farmacia nacional. Además de boticario, labor que ya no sumaba muchos créditos, se desempeñaba como profesor en el Instituto Nacional y había sido uno de los doce fundadores de la Facultad de Ciencias Matemáticas y Físicas de la Universidad de Chile en 1843, en donde se encargó del ámbito de la química. También participaba de la enseñanza de la farmacia en la Facultad de Medicina y asistía al Estado en distintas comisiones científicas como, por ejemplo, la comisión destinada a conseguir recursos para mejorar el recién fundado Gabinete de Historia Natural. ${ }^{30}$ Paralelamente, se fraguaba la primera organización de farmacéuticos, que se constituiría hacia 1858 como Sociedad de Farmacia, de la que Bustillos sería fundador y presidente. Por otra parte, Salinas representaba al boticario y al discípulo que en su doble identidad de informante y vendedor no solo fue llamado para apoyar al juez, sino también para participar en el juicio como testigo junto a los boticarios de la capital que debían declarar sobre su posible participación en la comercialización del veneno. ${ }^{31}$

Sin embargo, el llamado judicial no tuvo buena recepción. Bustillos declinó inicialmente informar al juez, dando cuenta en su negativa de la seriedad con que debía tratarse la pericia y las pocas garantías que ofrecían las condiciones de su ejercicio. Explicó que carecía de tiempo para un proceso que calificó como dificultoso y oneroso, y aprovechó para acusar las exigencias que acompañaban su identificación como experto. El formar parte de una élite científica tenía sus beneficios, pero también sus costos por las diversas tareas que se le encomendaban a diario, añadidas a sus responsabilidades universitarias. Así, en su respuesta inicial señaló:

Como debe saberlo US soy uno de los profesores del Instituto Nacional y los ramos de enseñanza que desempeño, me ocupan la mayor parte del día, cuya obligación no puedo desatender. A pesar de esto el Supremo Gobierno me ocupa frecuentemente en varias comisiones científicas, de las cuales tengo actualmente dos entre manos y una de ellas tengo cabalmente que evacuar entre hoy y mañana, quedándome otra por concluir. Por otra parte, estos cargos son demasiado onerosos y no es justo que se sumen muchos a la vez en un solo individuo; debiéndose distinguir las cargas públicas, si es que a más de las contribuciones públicas, estamos algunos obligados a [pagarlas] por nuestros conocimientos. ${ }^{32}$

de la farmacia que realizan análisis como para los "ensayadores generales" formados en el Instituto Nacional, en las escuelas de minas y, a partir de 1843, en la Universidad de Chile en ingeniería.Véase, Claudio Gutiérrez y Michael Reynolds, "Institucionalización y profesionalización de la Ingeniería en Chile”, Quipu. Revista Latinoamericana de Historia de las Ciencias y la Tecnología 16.1 (2014): 63-86.

30. Zenobio Saldivia, La ciencia en el Chile decimonónico (Santiago: Ediciones Universidad Tecnológica Metropolitana, 2005).

31. "Proceso indagatorio", f. $28 \mathrm{v}$.

32. "Proceso indagatorio", f. 12. 
Esta negativa — que no fue bien recibida por el juez, quien recordó la multa de 200 pesos asociados al rechazo de la petición - ${ }^{33}$ posiblemente se anudaba al reconocimiento de que las autoridades no entregaban a los peritos las condiciones necesarias para realizar su misión fármaco legal. En este sentido, el desprecio de Bustillos a los tribunales no reducía la relevancia que la justicia tenía para la farmacia, sino, más bien, podemos pensar que la misma relevancia otorgada a la química como herramienta de prueba, era lo que llevaba a exigir una mayor consideración. Bustillos como rector de la Sociedad de Farmacia reconoció en varias oportunidades que la farmacia debía "ilustrar" a las autoridades "en los casos de Farmacia Legal", "ocuparse del examen o análisis de los artículos de primera necesidad, que puedan comprometer la salud" y prestar "su cooperación en todo lo relativo a la higiene pública". ${ }^{34}$ En este contexto, en su negativa no solo intentaba conciliar el buen servicio público con sus otras responsabilidades profesionales, sino también negociar un trato más adecuado y justo por parte de los tribunales para su ciencia. Estas condiciones remitían a formalizar la pericia, a reducir su carácter discrecional y a convocar peritos preparados con conocimientos y capacidades en el desciframiento de la verdad judicial. ${ }^{35}$

Esta tensión entre la demanda por peritos y las condiciones de realización de la pericia recordaba las dificultades enfrentadas por la farmacia en la sociedad. Los representantes de la Sociedad de Farmacia denunciarían con posterioridad que, pese al aporte sustantivo que su ciencia ofrecía, esta no encontraba el respaldo que necesitaba. Así, si bien detallaban el valor de su gestión en los tribunales al vislumbrar un crimen oculto y develar una verdad escondida a los ojos de los comunes, también daban cuenta de cómo la falta de apoyo fragilizaba su misión y los exponía. ${ }^{36}$ La Sociedad, ofreciendo una instancia de unión y promoción de la profesión, negoció con el Estado las condiciones de la participación de la farmacia en tribunales ${ }^{37}$ y defendió la importancia de "ilustrar a la autoridad" en la farmacia legal. ${ }^{38}$ En una carta firmada por Bustillos, su presidente, y por ÁngelVásquez (18231897), su vicepresidente, ${ }^{39}$ se explicaba que el rechazo de algunos farmacéuticos a

33. La obligatoriedad de la pericia persistiría. En 1874 el Código Penal castigó al profesional que llamado como perito o testigo se negara a prestar declaración. Se le castigó con una pena de prisión en su grado medio o máximo de 21 a 60 días, o una multa entre 10 a 100 pesos. Véase Legislación chilena sobre Medicina Legal (Santiago: Imprenta Cervantes, 1907) 4.

34. Anales de la Sociedad de Farmacia de Santiago (Santiago) 1863: 9.

35. Anales de la Sociedad de Farmacia de Santiago (Santiago) 1863: 318.

36. Anales de la Sociedad de Farmacia de Santiago (Santiago) 1863:114-115.

37. La Sociedad estaba dirigida por Bustillos, presidente;Ángel SegundoVásquez, vicepresidente;Antonio León, secretario; Ruperto Romero, pro-secretario, y JoséV. Gutiérrez, tesorero. Entre los 41 miembros se contaba a médicos, botánicos y farmacéuticos, como Domeyko, Gay, Cox y Blest, entre otros. Guía de los encargados de la Policía Sanitaria en Chile (Valparaíso: Imprenta del Mercurio, 1868) 195.

38. Guía de los encargados 170.

39. Profesor de la cátedra de Química Orgánica y Farmacia, y miembro desde 1853 de la Facultad de Ciencias Matemáticas y Físicas en sucesión de Francisco García Huidobro.Véase Óscar Aedo, El sabio Ángel Vázquez (1823- 1893) (Santiago:Academia de Ciencias Farmacéuticas de Chile, 2000). 
participar en los trabajos periciales revelaba una situación preocupante: la falta de aparatos necesarios para la realización de análisis. El mensaje no desacreditaba el rol del farmacéutico en la pericia, sino, por el contrario, demandaba un cambio en las condiciones materiales de la química forense. Aludía a que esta situación no solo afectaba a los peritos, sino que complicaba al Estado al prolongar los juicios "pues los tribunales de justicia, i la autoridad municipal sin saber qué hacer, tienen que ir nombrando sucesivamente diferentes profesores" hasta encontrar quienes pudiesen verificarlos. ${ }^{40}$

La Sociedad defendió al farmacéutico e intentó posicionar su labor, y en esa defensa dio cuenta de la importancia de su quehacer "fármaco-legal". Distinguido por "su idoneidad i sus luces", por su "celo i su desinterés" y por su capacidad de ilustrar a los tribunales de justicia también se reconoció que para una adecuada realización de su misión el farmacéutico debía contar con un contexto que le permitiera desplegar las habilidades mencionadas y poner en práctica su capacidad científica. ${ }^{41}$ Era necesario un marco material que permitiera la implementación de su metodología y el uso de instrumentos. Así, uno de los primeros requerimientos levantados por la Sociedad de Farmacia, que iba más allá de la demanda forense, fue solicitar al erario nacional que apoyara la compra de los aparatos y enseres necesarios para la formación de un laboratorio para la asociación; se aprovechaba, además, que Claudio Gay estaba presto a partir a Europa y que se había ofrecido sin remuneración a adquirir los aparatos en el viejo continente. ${ }^{42}$ Pese a que contar con un laboratorio resultaba imperativo para complementar los limitados espacios de análisis oficiales existentes en el país y a través de ellos apoyar la labor pericial, recién en 1865 llegarían desde Europa los aparatos e instrumentos requeridos, pero como se indicaría en una misiva al Ministerio de Justicia "sin tener donde armarlos i colocarlos". 43

De vuelta al juicio de 1850, ante la posibilidad de una multa, Bustillos aceptó asistir al juez como lo había hecho en innumerables ocasiones y por más de veinte años. Si bien los farmacéuticos potencialmente capaces de participar en la pericia seguían siendo pocos, en las últimas dos décadas se habían formado más de una decena de farmacéuticos cuyo perfil los hacía aptos — algunos más, otros menospara el análisis forense. Los mismos expedientes judiciales de Santiago traslucen esta situación al presentar en la pericia a farmacéuticos conocidos, profesionales y vinculados a la docencia o a la Sociedad de Farmacia como los expertos más competentes para estas materias, a diferencia de lo ocurrido en provincia en donde la falta de farmacéuticos flexibilizaba la convocatoria.

Estas condiciones dan cuenta del reconocimiento de una experticia particular de los farmacéuticos en el ámbito forense. Al momento de convocar a especialistas

40. Anales de la Sociedad de Farmacia de Santiago (Santiago) 1863: 117

41. Anales de la Sociedad de Farmacia de Santiago (Santiago) 1863: 36.

42. Anales de la Sociedad de Farmacia de Santiago (Santiago) 1863:117.

43. Anales de la Sociedad de Farmacia de Santiago (Santiago) 1865: 342. 
para informar a la justicia, los jueces recurrieran a quienes parecían contar con las garantías suficientes para ofrecer una mirada experta. Se avalaba a los farmacéuticos en desmedro de los médicos, en un universo que, para la provincia de Santiago, y según el censo de 1865, alcanzaba ya 61 titulados en ejercicio y crecería sostenidamente en los próximos años. ${ }^{44}$

Bustillos como autoridad fundacional del área prescindió del título y contrarrestó esa ausencia con la experiencia acumulada. En el juicio de 1850 presentó junto con Salinas un reporte de dos carillas que siguió un formato compartido por la mayoría de los informes elaborados por farmacéuticos en tribunales a partir de mediados del siglo, con lo que evidenciaba nuevos modos de registro pericial impregnados por la farmacia y la formalización académica de su oficio. Este reporte iniciaba con la descripción física de los objetos enviados a análisis, y consideraba su soporte (un paquetito, un frasco, un disco de vidrio opaco), su contenido (una sustancia adherida de color blanco, un líquido viscoso, restos de medicamento, polvos blancos) y su estado (sellado y lacrado, sin cierre ni sello de ninguna clase). Luego seguía el análisis de las sustancias, su exposición a distintos contextos: al fuego, al agua y a otros reactivos, y la presentación de los resultados, que en ocasiones venían acompañados de alguna muestra que diera respaldo al hallazgo. En el reporte se describieron los métodos del análisis: la aplicación de carbones encendidos sobre los dulces y pasteles llevaron a que esta exhalara "olor a ajos", por su parte, el agua de cal y de barita generó unos "precipitados blancos" y el nitrato argéntico un "precipitado color teja". Estos procedimientos permitieron pensar una resolución, que fue confirmada con la aplicación del aparato de Marsh que generó el olor "fétido que caracteriza a la alcarcina" y permitió establecer la evidencia del carácter "eminentemente" tóxico del hallazgo, el cual resultó ser arsénico. ${ }^{45}$

Este informe reflejaba los progresos del área y contribuía a desplegar la especificidad de su conocimiento en el escenario antes descrito. Los resultados presentados por Bustillos representaban no solo un cumplimiento pericial, sino una exhibición del potencial de su ciencia como recurso capaz de contribuir, no sin dificultades, en el entramado burocrático judicial. Si bien, esta tarea pericial no fue el único espacio donde los farmacéuticos midieron y probaron su capacidad, además, representó un nicho atractivo y significativo para dar cuenta de parte de su quehacer. Su gestión permitió asignar o restar un carácter criminal a un acusado y determinar identidades en una serie de sustancias antes ilegibles. De la misma manera, representó una instancia de desarrollo del propio saber, en cuanto ejercicio de análisis que se hacía público y que, por tanto, debía no solo ser efectuado, sino

44. Censo jeneral de la República de Chile (Santiago: Imprenta Nacional, 1866) 182. A estos 61 se sumaban aquellos que ejercían de forma ilegal o que sin contar con título obtenían licencias para continuar desempeñándose como boticarios en sus establecimientos. Véase también Jacqueline Dussaillant Christie, “Turnos, títulos e 'intrusos': Los dolores de cabeza de los boticarios (Santiago, 1846-1943)”, Historia 48.1 (2015): 99-118.

45. "Proceso indagatorio", ff. 25r-25v. 
comunicado a través de la escritura y en ocasiones defendido a propósito de las controversias que surgían en el ejercicio científico. ${ }^{46}$

Así como la farmacia contribuyó en el desarrollo de la práctica química forense, esta última incidió en la conformación de un cuerpo teórico nacional de farmacia legal. Las experiencias judiciales de Bustillos y de sus contemporáneos servirían como fuente para sus publicaciones; eran citadas en los textos de la Sociedad de Farmacia, en las tesis y en los libros. ${ }^{47}$ Podemos pensar que esta experiencia forense fue significativa en los tiempos de Bustillos, tiempos de bisagra, en los que la farmacia comenzaba a alejarse de la "ignorancia en materia de ciencias naturales", cuando casi "no existían cursos públicos ni privados, pues faltaban los profesores, i aún los libros eran raros i dificiles de conseguir". ${ }^{48} \mathrm{Si}$ bien este aporte se entremezclaba con otros contextos de aprendizaje, la pericia ofreció y continuaría ofreciendo una instancia especial que sería tomada expresamente por la medicina legal a fines del siglo XIX, como dan cuenta los escritos de Federico Puga Borne, precursor del área. ${ }^{49}$ Estos textos adquirirían importancia para formalizar aspectos no regulados por la ley y para comunicar experiencias judiciales que, si bien no establecieron jurisprudencia, contribuyeron al desarrollo de una cultura forense nacional que sirvió como sustrato para el reconocimiento de su labor y para el apoyo de su gestión. ${ }^{50}$

El informe de Bustillos y Salinas, pese a la precariedad, confirmó el aporte pericial de la farmacia y de la química, y el saber y el oficio de los informantes. Los farmacéuticos comenzaron a ser identificados como los únicos capaces de dar una respuesta desde la ciencia a crímenes notorios que requiriesen de análisis químicos con los que se ofrecía orden en el desorden y comprensión en la opacidad. Durante este periodo gran parte de los peritos convocados por la justicia pertenecieron a la Sociedad de Farmacia y correspondieron a farmacéuticos titulados, si bien aún existían algunos integrantes mayores, como Bustillos, que no contaban con una formación oficial y también algunos que provenían de otras disciplinas. Sus miembros fueron convocados frecuentemente, a solicitud del juez o de Bustillos, quién como presidente de la agrupación estuvo encargado de proponer las comisiones más apropiadas para Santiago. ${ }^{51}$ En esta dinámica, el presidente de la Sociedad se

46. Respecto al potencial de las controversias, véase José Ramón Bertomeu y Ximo Guillem-Llobat, "Following Poisons in Society and Culture (1800-2000): A Review of Current Literature", Actes d'Història de la Ciència i de la Têcnica 9 (2016): 13.

47. Bustillos describiría, por ejemplo, la aplicación de métodos de detección más precisos, como el método de Marsh basado en la reducción de arsénico descrito en 1836 por James Marsh en Londres. Véase José Vicente Bustillos, Elementos de farmacia aplicada a la medicina (Santiago: Imprenta del Ferrocarril, 1856).

48. Torres 285 .

49. Puga Borne 490-491.

50. Por ejemplo, las dos publicaciones del Instituto de Higiene, El Boletín de Hijiene i Demografía (1898-1921) y la Revista Chilena de Hijiene (1894-1914), junto a la variedad de textos, manuales y estudios de higiene y medicina legal.

51. Las publicaciones de la Sociedad de Farmacia dan cuenta de estas demandas, por ejemplo, Anales de la Sociedad de Farmacia de Santiago (Santiago) 1863:366-369; Anales de la Sociedad de Farmacia 
instaló como intermediario entre el perito y el juez. De este modo, remitía el informe y entregaba una lectura sumaria que reforzaba el papel mediador y legitimador de la agrupación. En ocasiones este papel se proyectó más allá de los juzgados por iniciativa de los propios farmacéuticos, como cuando a propósito de un análisis toxicológico realizado en 1863 en un caso de envenenamiento, como presidente de la Sociedad, Bustillos escribió al intendente recomendándole se prohibiera la confección de confites coloreados por su carácter venenoso. ${ }^{52}$ Así también lo hizo en 1864 cuando Ángel Segundo Vázquez encontró un veneno abortivo en una sustancia, lo que llevó a Bustillos a recordar a las autoridades sobre "el abuso de la venta de artículos venenosos" y la necesidad de "poner coto a ellos" ${ }^{53}$ En este sentido, la proyección de la acción pericial a otros ámbitos de la sociedad se relacionaba con el reconocimiento que ellos mismos hicieron a su capacidad para identificar riesgos tóxicos. ${ }^{54}$ Esta atribución sería legitimada por las autoridades por medio de diversas regulaciones que apuntaban a ejercer control con el análisis en laboratorio, realizado por farmacéuticos, sobre la calidad de dichos productos.

El arribo de la Sociedad de Farmacia a tribunales dio cuenta del diálogo entre los hechos químicos, los argumentos legales y las experiencias judiciales mediado ya no solo por conocedores que comenzaron a ser reconocidos por su saber particular, sino por expertos certificados y colectivos profesionales que ayudaron a definir dicha experticia. Para 1850 los médicos habían sido desplazados por los farmacéuticos como peritos, ${ }^{55}$ pese a ello, las carencias de los propios farmacéuticos y la diversificación de su disciplina introducirían en la discusión a un nuevo actor: el toxicólogo, que comenzó a ser solicitado por las autoridades hacia fines de la década de 1860.

\section{Lemetayer y el Instituto de Higiene en tribunales, 1897}

El 25 de enero de 1897 Ángela Von Dessare fallecía en su domicilio tras haber ingerido sublimado. ${ }^{56}$ Si bien había dejado una carta informando un acto suicida, la justicia abrió un sumario destinado a descartar la participación de terceros y a confirmar el carácter de las sustancias consumidas, proceso que llevó a que el cadáver

de Santiago (Santiago) 1864: 89-91.

52. Anales de la Sociedad de Farmacia de Santiago (Santiago) 1864: 91-92.

53. Anales de la Sociedad de Farmacia de Santiago 1864: 332.

54. Véase María José Correa Gómez, “Temor y atracción. El veneno en la imaginación urbana. Chile, 1880-1920”, História Unisinos 23.2 (2019): 167-179.

55. El curso de Química Fisiológica y Patológica el segundo año de Medicina solo entregaba una aproximación inicial al análisis toxicológico. En sexto año estos temas eran revisitados en el curso de Medicina Legal, el cual consideraba contenidos de toxicología.

56. El sublimado había sido adquirido en la botica del señor Elías Villarroel en Independencia, esquina Avenida del Rosario. "Suicidio de Anjela Von Dessarre", Santiago, 1897. ANH, Santiago, Archivo Judicial de Santiago, caja 1171, exp. 5. 
fuese remitido a la morgue para su reconocimiento médico, y las vísceras junto al líquido medicamentoso al Instituto de Higiene para su análisis.

Para 1897 existía una regulación más definida que apoyada en nuevas instituciones contribuyó a reafirmar la ciencia en la pericia. Esta tendencia se afianzó con la promulgación de los códigos civil y penal, para luego reforzarse con sus respectivos códigos de procedimiento, los cuales determinaron que los análisis químicos debían ser ordenados solo por el juez y cuando fuese indispensable, y ser realizados en "los laboratorios fiscales o municipales". ${ }^{57}$ Estos informes debían estar escritos y contener, al menos, "la descripción de la persona o cosa que fuere objeto de él, del estado i del modo en que se hallare", la relación "de todas las operaciones practicadas i de su resultado" y las conclusiones "conforme a los principios o reglas de su ciencia, arte u oficio", 58

En el Congreso, los parlamentarios insistieron en sus discusiones sobre el fomento de las ciencias ante la necesidad de contar con farmacéuticos titulados no solo para que las boticas fuesen regentadas por profesionales, sino para que la justicia pudiese descansar en sus aportes. La preocupación por el desarrollo de la toxicología comenzó a manifestarse hacia 1870 en un contexto nacional que se reconocía falto de profesores de estas materias. “¿Podría decirme el señor Ministro" - le preguntarían en la Cámara a Miguel Luis Amunátegui (1828-1888), ministro de Instrucción Pública - si entre los cinco médicos enviados a Europa en 1878 había alguno que se hubiese dedicado "al estudio especial de la toxicología?". ${ }^{59}$ La interrogación nacía del reconocimiento de la importancia de contar con personas que auxiliaran a la justicia en la investigación de los envenenamientos y orientaran en estas materias. ${ }^{60}$ ¿Qué clase de auxilio científico puede prestar a la justicia un farmacéutico recibido? Un individuo que no ha estudiado química ¿Cómo sabrá si en una muerte ha habido, por ejemplo, envenenamiento?”, se preguntaban algunos parlamentarios hacia 1877, con lo que daban cuenta de la importancia que comenzaba a adquirir el tema del análisis y su aplicación en la justicia. ${ }^{61}$

Para fin de siglo, la creación del Instituto de Higiene (1892) y el desarrollo de la medicina legal ayudó a otorgar mayor protagonismo a la toxicología y a sus expertos como intérpretes de los cuerpos y las sustancias sospechosas remitidas por los jueces. El primero ofreció un espacio formal para el análisis, la Sección

57. Bernardino Bravo, "Los comienzos de la codificación en Chile: la codificación procesal", Revista Chilena de Historia del Derecho 9 (1983): 194. En 1906 el Código de Procedimiento Penal estableció que los jueces debían pedir informe de peritos solo en casos determinados por la ley y siempre que la apreciación del hecho o de la circunstancia obligase "conocimientos especiales de alguna ciencia, arte u oficio". Código de Procedimiento Penal de la República de Chile (Santiago: Imprenta Cervantes, 1906) 84, 91.

58. Código de Procedimiento Penal 89.

59. Diario Oficial (Santiago) 7 de enero de 1878.

60. La toxicología aparece por primera vez en el diccionario de la Real Academia Española en 1884. Era definida como parte de la medicina que trata de los venenos.

61. Diario Oficial (Santiago) 8 de noviembre de 1877. 
de Química y Toxicología, ${ }^{62}$ la que a su vez contaba con una subsección de toxicología a cargo del jefe de la Sección de Química que, si bien no fue prevista en la ley de creación del Instituto, comenzó a operar desde los inicios del establecimiento como resultado de las necesidades de la administración judicial del país. ${ }^{63}$ La sección correspondió entonces al primer espacio reconocido explícita y específicamente para dicha función, y estuvo dirigido por Pedro Lemetayer. Si bien para 1897 el Instituto contaba con solo cinco años de creación y daba cuenta de dificultades en su gestión, rápidamente se perfiló como el principal sitio de análisis toxicológicos. Recibió muestras de la capital y de los varios tribunales de provincia que existían en el país, con cifras constantes, como resume la Tabla 1 sobre el movimiento del Instituto, lo que persistiría hasta la creación del Instituto Médico Legal en la década de 1920.

Tabla 1. Movimiento de los servicios del Instituto de Higiene entre 1898 y 1907

\begin{tabular}{|c|c|c|c|c|c|c|c|c|c|c|}
\hline Análisis / años & 1898 & 1899 & 1900 & 1901 & 1902 & 1903 & 1904 & 1905 & 1906 & 1907 \\
\hline $\begin{array}{c}\text { Informes clínicos } \\
\text { industriales, aguas potables } \\
\text { y termales }\end{array}$ & 569 & 1356 & 1406 & 1343 & 1345 & 1285 & 1045 & 1044 & 1500 & 1535 \\
\hline $\begin{array}{l}\text { Informes a los juzgados } \\
\text { (toxicológicos) }\end{array}$ & 44 & 34 & 31 & 29 & 36 & 3 & 33 & 47 & 34 & 29 \\
\hline $\begin{array}{c}\text { Notas e informes al } \\
\text { gobierno }\end{array}$ & 34 & 51 & 44 & 44 & 30 & 49 & 53 & 67 & 58 & 103 \\
\hline $\begin{array}{c}\text { Notas a intendentes y } \\
\text { gobernadores }\end{array}$ & 103 & 129 & 142 & 132 & 170 & 124 & 123 & 114 & 24 & 111 \\
\hline $\begin{array}{c}\text { Al Consejo Superior de } \\
\text { Higiene }\end{array}$ & 13 & 55 & 57 & 57 & 60 & 66 & 71 & 764 & 71 & 26 \\
\hline $\begin{array}{l}\text { A los alcaldes y otras } \\
\text { corporaciones }\end{array}$ & 222 & 453 & 405 & 369 & 325 & 261 & 261 & 271 & 118 & 129 \\
\hline
\end{tabular}

Lemetayer fue quien recibió del juzgado las vísceras de Ángela Von Dessare y los restos de la poción bebida antes de su muerte para su análisis, material para pruebas que ingresó al registro de su sección bajo el número 88. Como consta en otros procedimientos periciales, estas eran remitidas al juez por el médico de ciudad, cuando sospechaba de un envenenamiento tras la realización de la autopsia, lo

62. El Instituto contó, además, con secciones de Microscopía y Bacteriología y de Higiene y Estadística, a las que se sumaron luego las de Seroterapia e Instituto de Vacuna Animal y el Desinfectorio Público. Véase Pedro Lautaro Ferrer, Álbum gráfico del Instituto de Higiene de Santiago (Santiago: Instituto de Higiene Pública, 1910).

63. Ricardo Dávila, Hijiene pública en Chile (Santiago: Imprenta Cervantes, 1908). Tras la creación del Instituto de Higiene comenzaron a surgir laboratorios municipales orientados al examen de las sustancias alimenticias y de las bebidas, que en ocasiones complementaron la labor del Instituto. 
que sugería la realización de un examen toxicológico. ${ }^{64}$ Una vez en el Instituto, las muestras eran estudiadas y sus conclusiones enviadas al juzgado, en muchas ocasiones con ejemplos de los hallazgos obtenidos en el laboratorio, como aquel "tubo cerrado" con "parte del análisis del sulfuro de arsénico obtenido" socializado por Lemetayer en un caso de envenenamiento por arsénico ocurrido en Santiago en 1893, lo que sirvió como prueba y "pieza de convicción” de los resultados. ${ }^{65}$ En el caso de Ángela, Lemetayer estableció que los restos orgánicos contenían mercurio en una cantidad letal, y que la poción medicamentosa correspondía a bicloruro de mercurio (sublimado) disuelto en alcohol. ${ }^{66}$ Esta indagación fue un práctica habitual que, si bien no alcanzó grandes proporciones, como se refleja en la imagen relacionada con el movimiento de los servicios del Instituto, llevó a la constatación del carácter venenoso de una gran variedad de sustancias que solían contener o ser, para fines de siglo, estricnina, cloral, mercurio, ácido oxálico y morfina. ${ }^{67}$

La colección de piezas médico-legales presentadas en el Compendio de Medicina Legal de Puga Borne entrega un retrato perspicaz del toxicólogo como experto en el manejo de la química y personaje clave en el desenvolvimiento del juicio. Publicado después de la inauguración del Instituto, contiene casos nacionales (e internacionales) que relatan en tono heroico los desafios enfrentados por los toxicólogos, mientras que muestran el impacto de su quehacer en la justicia, recalifican a través del análisis enfermedades, accidentes y homicidios como envenenamientos criminales o no intencionales. En este sentido, textos como estos, fundantes y en cierto modo épicos, reflejaron un modelo pericial que pese a alejarse del cotidiano forense, marcado por conflictos, proyectaron varios de los espacios ganados por la química a través de la figura de los toxicólogos.

Estas historias judiciales, sea la colección de historias médico legales insertas en el manual de Puga Borne, las comunicadas en la prensa o las registradas en los archivos, contribuyeron a dibujar el escenario pericial ideal a alcanzar a fin de siglo. Estos registros compartieron, de distintas maneras, las tres etapas a seguir en tribunales: revisión de los síntomas provocados por el presunto veneno, exploración de las lesiones anatómicas reveladas por la autopsia y reconocimiento del veneno por medio del análisis químico, esta última a cargo del toxicólogo. ${ }^{6}$

Lemetayer apareció en muchas de estas historia judiciales como el especialista capaz de develar significados y definir identidades por medio del manejo de tecnologías específicas y como autoridad en el ámbito de las "observaciones i ensayes preliminares" que requería la justicia. ${ }^{69}$ Midió pociones y las contrastó con las calidades de productos similares comercializados en boticas y droguerías de la capital,

64. "Suicidio de Anjela Von Dessarre", f. 6.

65. Puga Borne 745.

66. "Suicidio de Anjela Von Dessarre", f. 9.

67. Puga Borne 458.

68. Puga Borne 450.

69. Puga Borne 466. 
con lo que estableció errores en la conformación de las recetas médicas y cuestionó las prácticas de consumo de medicamentos y drogas. Su quehacer derivaba del reconocimiento institucional de las experticias y del desarrollo de procesos que contribuyeron a demarcar el quehacer del experto y de sus protocolos ante la justicia. Se buscaba racionalizar el análisis, asegurándose, entre otras cosas, que las muestras no llegasen al laboratorio contaminadas para no producir "complicaciones mui fastidiosas" en su estudio. Estas debían ser guardadas y trasladadas del juzgado al Instituto en "frascos de vidrios grandes i nuevos", conservados a baja temperatura, sellados con corcho, papel y lacre. Se debía añadir una etiqueta que presentara el contenido junto con la firma del médico y de los agentes judiciales que habían asistido a la autopsia.

Para fines del siglo XIX la botica o la participación en alguna sociedad de farmacia ya no resultaban suficientes para reconocer y atribuir capacidad específica en el ámbito de la química forense en procesos judiciales de envenenamiento. Se requería de otras trayectorias y pertenencias, identidades y alianzas, para respaldar el saber adquirido y la experiencia conquistada, que consideraban, en primer lugar, un vínculo con la institucionalidad higiénica y médico legal de preferencia radicada en el Instituto de Higiene. Por este motivo no resulta extraño los vínculos que los toxicólogos tuvieron con el Instituto. Compartieron una biografia asociada al laboratorio y mostraron cierta cercanía con el establecimiento. Varios de los que aparecen participando como expertos en los juicios fueron ayudantes del laboratorio o participaron en los concursos para entrar al establecimiento.

El caso de Pablo Marteens, quien fue convocado varias veces como perito en casos de envenenamiento, es ilustrativo. Farmacéutico, ayudante de la clase de Química General y Docimasia de la sección universitaria, participó en 1892 sin éxito en la terna para elegir al jefe de la Sección de Química del Instituto de Higiene, enfrentándose con Lemetayer. En 1893 concursó por el puesto de profesor del curso de Química Orgánica y, posteriormente, por el cargo de ayudante en la Sección de Química del Instituto de Higiene sin lograr los cupos. ${ }^{70}$ Sin haber entrado al Instituto, Marteens fue nombrado en 1898 profesor de ciencias físicas y naturales del Liceo de Iquique, lo que se entendió como una promoción. ${ }^{71}$ Posteriormente, se hizo cargo del Laboratorio Especial de Química de Iquique, donde junto con abocarse a realizar análisis, principalmente ensayos de productos mineros (cobre, azufre, plata, oro, salitre, boratos y sales potásicas), coordinó la adquisición de materiales desde Europa y realizó clases teóricas y prácticas para jóvenes enviados por productores y por administradores de oficinas salitreras que necesitaban ensayadores para el estudio de sus productos. ${ }^{72}$ En esa ciudad y lejos de la capital, los conocimientos de Marteens fueron aplaudidos y requeridos junto al

70. Diario Oficial (Santiago) 17 de noviembre de 1893; Diario Oficial (Santiago) 30 de abril de 1894.

71. Diario Oficial (Santiago) 30 de abril de 1898.

72. Durante los primeros meses se hicieron 393 ensayos de minerales y se analizaron fuentes de agua de Iquique. En las primeras clases se inscribieron 18 alumnos, con asistencia de 15. 
de otros hombres que realizaban también labores de análisis como Amador Guajardo, químico municipal de la ciudad de Iquique y, con anterioridad, ayudante de la Sección de Química del Instituto de Higiene. En cierto sentido constituían un trozo de la institucionalidad higiénica de la capital, y expertos posibles de aportar en el desarrollo del análisis y de la toxicología en la provincia.

Junto a Marteens y Guajardo surgieron más nombres como el de Aurelio Zilleruelo, ayudante del Laboratorio Químico Municipal de Valparaíso, o Rufino Concha, ayudante de la clase de Química Orgánica y Farmacia de la Universidad de Chile y luego de la Sección de Química del Instituto de Higiene hacia 1895. Sus biografias retratan el perfil del perito. Farmacéuticos titulados con conocimientos de toxicología y una trayectoria laboral vinculada al análisis; se diferenciaban de aquellos farmacéuticos titulados dedicados al trabajo en boticas. La formación en el extranjero aún no estaba consolidada para este grupo. El mismo Consejo Superior de Higiene Pública había insistido en varias oportunidades sobre la "necesidad de enviar a uno de los empleados de la sección de quimica y toxicólogia del Instituto de Hijiene a Europa, para que estudiara especialmente este último ramo", dado que eran "rarísimas" las personas que "se dedican a estudiar detalladamente los difíciles y graves problemas del análisis toxicolójico" tanto en Chile como en el extranjero. ${ }^{73}$ Carlos Ghigliotto fue uno de los primeros enviados a Europa para seguir estudios de toxicología, y fue contratado posteriormente como profesor del curso de Química Analítica y Botánica. ${ }^{74}$ En 1900 Rufino Concha viajaría al extranjero para estudiar Inspección Sanitaria y Química aplicada a la higiene, junto al doctor Luis Middleton.

Sus recorridos muestran el protagonismo e importancia del Instituto, en términos prácticos y simbólicos, como epicentro de la toxicología y como destino deseado, al mismo tiempo que documenta la existencia de otros espacios que participaron en la conformación de una experticia toxicológica — como laboratorios municipales, espacios universitarios y establecimientos aduaneros o escolares - ${ }^{75}$ El propio Lemetayer, antes de estar a cargo de la Sección de Química y Toxicología del Instituto, había sido director del Laboratorio de la Estación Agronómica de la Quinta Normal y durante sus primeros años como director de la Sección de Química usaría las instalaciones de la Estación Agronómica para hacer los análisis por no haberse inaugurado aún el edificio del Instituto.

Pese a esta diversidad, podemos plantear que los toxicólogos profundizaron su experiencia científica como burócratas de la administración pública y contribuyeron a la institucionalización de la química como recurso del Estado. Esta vinculación da cuenta de una institucionalización de la pericia, que pasa de una gestación autónoma, asociada al prestigio de boticarios y profesores o de colectivos

73. Diario Oficial (Santiago) 30 de mayo de 1896.

74. Diario Oficial (Santiago) 4 de mayo de 1896. Si bien en 1862 el gobierno había apoyado el viaje de Carlos Middleton a Europa para estudiar Química Analítica, Ghigliotto correspondió a uno de los primeros enviados bajo el espíritu de ofrecer becas permanentes a jóvenes profesionales.

75. Diario Oficial (Santiago) 25 de abril de 1895. 
profesionales, a una creación institucional. Esto explica, por ejemplo, que estuviese asociada en algunos casos a dos nombres, el del toxicólogo que realizaba el análisis y el del director de la institución que informaba y avalaba los resultados. En el juicio de Ángela, Lemetayer firmó el análisis acompañado por una misiva del doctor Alejandro del Rio, quien como autoridad máxima resumió y respaldó el estudio en una lógica similar a la que comenzaba a mostrarse en algunos casos en los que participó Bustillos como presidente de la Sociedad de Farmacia.

\section{Conclusiones}

La pericia permite observar la ciencia más allá del laboratorio en diálogo con su conformación universitaria, con su función pública y como recurso de la burocracia estatal. Permite ver a sus promotores en su formación como especialistas, en su relación con las instituciones y en sus compromisos disciplinares, en operación como uno de los varios lentes para acceder a procesos que contribuyeron al desarrollo del saber químico.Y es este universo que enmarca al experto y a su ciencia el que le otorga un contexto sociopolítico que colaboró en su afirmación y delimitación profesional y científica. Es entender que, si bien la ciencia se presentaba a sí misma como un saber fáctico, con una identidad objetiva que desconocía los avatares hermenéuticos, su activación en tribunales supuso el despliegue de su subjetividad y de su carácter interpretativo. Estas condiciones acercaron el acto pericial a la lógica judicial con lo que ubicaron a los peritos como sujetos que interactuaban fuertemente con las dinámicas y condiciones ofrecidas por la justicia desde sus formaciones, pertenencias y saberes.

Hacia 1830, en un momento en el cual la justicia no oficializaba la identidad de este tipo de perito y en el que la universidad tampoco reconocía la especialización en estas materias forenses, podemos plantear que estas pericias representaron una tribuna importante para los profesionales de la química, así como para su vinculación con la farmacia y su conformación como especialistas en la práctica del análisis. Los tribunales de justicia representaron estrados atractivos para dar a conocer estos quehaceres, enseñar el potencial del laboratorio y sus procesos y otorgar un mayor protagonismo a la disciplina de la farmacia y a sus cultores.

Los convocados para la pericia respondieron a los criterios de experticia vigentes en esos años. Primero, boticarios sin título reconocidos por su aporte a una sociedad que transitaba del naturalismo a la ciencia, seguidos por farmacéuticos titulados, formados en el programa de tres años que ofreció el Instituto Nacional entre 1833 y 1842, o en los estudios de farmacia que entregó la Facultad de Medicina a partir de 1843, y agrupados desde mediados del XIX en la Sociedad de Farmacia. Y, finalmente, farmacéuticos, u otros científicos, que sumaron a sus credenciales universitarias un conocimiento específico en toxicología, adquirido no tanto en sus estudios, sino en su participación en la burocracia estatal y, en específico, en las labores de análisis que se efectuaron en laboratorios fiscales, espacios educacionales y aduanas. 
La creación y presentación de evidencias en casos de envenenamiento obligó a los farmacéuticos a desarrollar un ejercicio comunicativo complejo, anudado a la transformación de un hecho químico en un hecho judicial que se manifestaba a través de un escrito marcado por su interpretación y direccionalidad. ${ }^{76}$ Esta exigencia requirió abonar a sus credenciales signos biográficos, institucionales, materiales, espaciales y retóricos para cimentar una experticia en ciernes. El tránsito del boticario al toxicólogo exigió no solo de título, sino experiencias demostrables y transmisibles. Demandó una mayor abstracción no solo en la capacidad para realizar el acto científico, sino para identificar, interpretar y comunicar un proceso, definir el análisis, explicarlo y comprenderlo teóricamente. Este acto de racionalización y síntesis implicó un control mayor del contexto institucional de su práctica y la conformación de grupos que compartieron espacios intelectuales y sociales dentro y fuera de la química y de los tribunales enmarcados, en parte, bajo la sombra del Instituto de Higiene. En este recorrido quedan muchas preguntas abiertas y ámbitos que explorar. La práctica del análisis, la cultura material científica, el laboratorio, el crimen y su repercusión, la incidencia en la misma justicia y la relación con los legos y con otros profesionales quedan como algunos de los temas pendientes en la configuración del experto en química desde tribunales.

\section{Fuentes}

\section{Manuscritas}

Archivo Nacional Histórico, Santiago (ANH)

Archivo Judicial de Santiago

\section{Impresas}

Barros Arana, Diego. Estudios histórico-bibliográficos. Santiago: Imprenta Cervantes, 1909-1911.

Bustillos, José Vicente. Elementos de farmacia aplicada a la medicina. Santiago: Imprenta del Ferrocarril, 1856.

Censo jeneral de la República de Chile levantado el 19 de abril de 1865. Santiago: Imprenta Nacional, 1866.

Código de Procedimiento Penal de la República de Chile. Santiago: Imprenta Cervantes, 1906. Graham, María. Diario de su residencia en Chile (1822) y de su viaje al Brasil (1823). Madrid: Editorial Americana, 1964.

Guía de los encargados de la Policía Sanitaria en Chile. Valparaíso: Imprenta del Mercurio, 1868.

76. Ian Burney, Poison, Detection, and the Victorian Imagination (Manchester: Manchester University Press, 2006) 79. 
Legislación chilena sobre Medicina Legal. Santiago: Imprenta Cervantes, 1907.

Puga Borne, Federico. Compendio de Medicina Legal adaptado a la legislación chilena. Santiago: Imprenta Cervantes, 1896.

Urizar, Fernando. Repertorio chileno. Santiago: Imprenta Araucana, 1835.

Ferrer, Pedro Lautaro. Álbum gráfico del Instituto de Higiene de Santiago. Santiago: Instituto de Higiene Pública, 1910.

Dávila, Ricardo. Hijiene pública en Chile. Santiago: Imprenta Cervantes, 1908.

\section{Periódicos y revistas}

Anales de la Sociedad de Farmacia de Santiago (Santiago) 1863-1865.

Anales de la Universidad de Chile (Santiago) 1874.

Diario Oficial (Santiago) 1877-1898.

\section{Bibliografía}

Aedo, Óscar. El sabio Ángel Vázquez (1823- 1893). Santiago: Academia de Ciencias Farmacéuticas de Chile, 2000.

- José Vicente Bustillos: padre de la farmacia chilena. Santiago: Academia de Ciencias Farmacéuticas de Chile, 1987.

Ariza, Juan Sebastián. “¿Remedios o ponzoñas? Aproximación al uso de la yerbatería como método curativo en el Nuevo Reino de Granada durante el siglo XVIII". Anuario de Historia Regional y de las Fronteras 19.2 (2014): 315-333. . "La cocina de los venenos. Aspectos de la criminalidad en el Nuevo Reino de Granada, siglos XVII-XVIII". Tesis de pregrado en Historia, Universidad del Rosario, 2013.

Ash, Eric. "Introduction: Expertise and the Early Modern State". Osiris 25.1 (2010): 1-24.

Bertomeu, José Ramón y Ximo Guillem-Llobat. "Following Poisons in Society and Culture (1800-2000): A Review of Current Literature”. Actes d'Història de la Ciència i de la Têcnica 9 (2016): 9-36.

Bohoslavsky, Ernesto y Germán Soprano. Eds. Un Estado con rostro humano. Funcionarios e instituciones estatales en Argentina (desde 1880 a la actualidad). Buenos Aires: Prometeo Libros, 2010.

Brangier, Víctor. "La fragilidad de la simbiosis médico-judicial y la producción de una antropología criminal. Juzgados del Crimen de Santiago, 18741906". Tesis de maestría en Historia, Universidad de Chile, 2008.

Bravo, Bernardino. "Los comienzos de la codificación en Chile: la codificación procesal". Revista Chilena de Historia del Derecho 9 (1983): 191-210.

Burney, Ian. Poison, Detection, and the Victorian Imagination. Manchester: Manchester University Press, 2006.

Correa Gómez, María José. “Temor y atracción. El veneno en la imaginación urbana. Chile, 1880-1920”. História Unisinos 23.2 (2019): 167-179. 
. "De la Casa de Orates al Juzgado. Pericia alienista y evaluación judicial de la locura en Santiago de Chile hacia 1860". História, Ciências, Saúde Manguinhos 20.2 (2013): 571-585.

Dussaillant Christie, Jacqueline. “Turnos, títulos e 'intrusos': Los dolores de cabeza de los boticarios (Santiago, 1846-1943)”. Historia 48.1 (2015): 99-118.

Eigen, Joel Peter. Witnessing Insanity. Madness and Mad-Doctors in the English Court. New Haven:Yale University Press, 1995.

Fabregat, Mario. “Justicia y procedimientos judiciales por suicidio e intento de suicidio. Chile: 1820-1920". Tesis doctoral en Historia, Universidad de Chile, 2017.

Franulic, Fernando. “Deber entrar a medicinarse...': la casa, el adentro y la purificación. El Hospital San Francisco de Borja entre 1830 y 1870”. Tesis de maestría en Historia, Universidad de Chile, 2007.

Golinski, Jan. Making Natural Knowledge. Constructivism and the History of Science. Chicago:The University of Chicago Press, 2005.

. Science as Public Culture. Chemistry and Enlightenment in Britain, 17601820. Cambridge: Cambridge University Press, 1999.

González, Ricardo. "Itinerarios de la profesión médica y sus saberes de Estado. Buenos Aires, 1850-1910". Los saberes del Estado. Coords. Mariano Ben Plotkin y Eduardo Zimmermann. Buenos Aires: Edhasa, 2012.

Gutiérrez, Claudio y Michael Reynolds. "Institucionalización y profesionalización de la Ingeniería en Chile”. Quipu. Revista Latinoamericana de Historia de las Ciencias y la Tecnología 16.1 (2014): 63-86.

Laval, Enrique. Botica de los jesuitas de Santiago. Santiago: Asociación Chilena de Asistencia Social, 1953.

Plotkin, Mariano Ben y Eduardo Zimmermann. "Introducción. Saberes de Estado en la Argentina, siglos XIX y XX”. Los saberes del Estado. Coords. Mariano Ben Plotkin y Eduardo Zimmermann. Buenos Aires: Edhasa, 2012.

Ronderos, Paula. "El arte de boticario durante la primera mitad del siglo XVII en el Nuevo Reino de Granada”. Fronteras de la Historia 12 (2007): 175-196.

Ruperthuz Honorato, Mariano y Marcelo Sánchez Delgado. "Entre la degeneración y el psicoanálisis: una pericia médico legal chilena en 1915”. Revista Historia y Justicia 4 (2015): 138-168.

Saldivia, Zenobio. La ciencia en el Chile decimonónico. Santiago: Universidad Tecnológica Metropolitana, 2005.

Sanhueza, Carlos. "Objetos naturales en movimiento. Acerca de la formación de las colecciones del Museo Nacional de Chile (1853-1897)". Revista de Humanidades 34 (2016): 143-169.

- Geografía en acción. Práctica disciplinaria de Hans Steffen en Chile (18891913). Santiago: Editorial Universitaria, 2014.

Sanhueza, Carlos. "El objetivo del Instituto Pedagógico no es el de formar geógrafos. Hans Steffen y la transferencia del saber geográfico alemán a Chile. 
1893-1907”. Historia 45.1 (2012): 171-197.

Schell, Patience. The Sociable Sciences. Darwin and His Contemporaries in Chile. New York: Palgrave Macmillan, 2013.

Shapin, Steven. Never Pure. Historical Studies of Science as if It Was Produced by People with Bodies, Situated in Time, Space, Culture, and Society, and Struggling for Credibility and Authority. Baltimore:The John Hopkins University Press, 2010.

Smith, Roger. Trial by Medicine. Insanity and Responsibility in Victorian Trial. Edinburgh: Edinburgh University Press, 1981.

Vetö, Silvana. "Homosexualidad masculina y examinación médica en la Casa de Menores de Santiago, 1929-1942”. Revista Historia y Justicia 10 (2018): 42-76.

Walker, Nigel. Crime and Insanity in England. Edinburgh: University Press Edinburgh, 1968. 